\title{
Efficacité du rechargement de la plage de Pilote Bar (Saint-Louis) après une décennie d'érosion
}

\author{
Omar Balde \\ Laboratoire de Sédimentologie et de Biostratigraphie, \\ Université Cheikh Anta Diop de Dakar, Sénégal \\ Dr. Issa Sakho
}

UMR Sciences Technologies Avancées et Développement Durable (UMR STADD), Université Amadou Moctar Mbow de Dakar, Sénégal, Dakar, Sénégal. UMR-CNRS, Morpho dynamique continentale et côtière, Université de Rouen Normandie, Cedex France

\section{Pr. Mouhamadoul Bachir Diouf}

Laboratoire de Sédimentologie et de Biostratigraphie, Université Cheikh Anta Diop de Dakar, Sénégal

\section{Dr. Mamadou Bop}

Laboratoire Eau, Energie, Environnement et procédés industriels (LE3PI), Ecole Supérieure Polytechnique (ESP), Université Cheikh Anta Diop de

\section{Dakar, Sénégal}

\section{Djibril Tine}

Laboratoire de Télédétection Appliquée de Dakar (LTA), Institut des

Sciences de la Terre, Université Cheikh Anta Diop de Dakar, Sénégal

\section{Wardou Ibouroi}

Laboratoire de Sédimentologie et de Biostratigraphie, Université Cheikh Anta Diop de Dakar, Sénégal

\section{Doi:10.19044/esj.2021.v17n10p207}

Submitted: 30 November 2020

Accepted: 23 February 2021

Published: 31 March 2021

\author{
Copyright 2021 Author(s) \\ Under Creative Commons BY-NC-ND \\ 4.0 OPEN ACCESS
}

\section{Cite As:}

Balde O., Sakho I., Diouf B. M., Bop M., Tine D. \& Ibouroi W. (2021). Efficacité du rechargement de la plage de Pilote Bar (Saint-Louis) après une décennie d'érosion. European Scientific Journal, ESJ, 17(10), 207. https://doi.org/10.19044/esj.2021.v17n10p207

\section{Résumé}

L'ouverture d'une brèche dans la bande de sable de la Langue de Barbarie à Saint Louis (Sénégal) en 2004 a eu des conséquences sur l'étendue du bief fleuve-mer du Gandiolais. En effet, les zones en face de la brèche 
deviennent très vulnérables à l'action des vagues dévastatrices de la Grande Côte. La décision d'une protection douce par ensablement a été prise pour protéger le village de Pilote Bar des risques d'érosion et de submersion marine. Cette première expérience au Sénégal a donné des résultats positifs. Le rechargement de la plage de Pilote Bar a favorisé la formation d'une lagune. Une sédimentation et une augmentation de la hauteur des pentes des plages voisines ont également été observées. Les vitesses de courant mesurées par l'ADCP RIVER $1200 \mathrm{~kW}$, présentent un fonctionnement normal caractéristique d'une zone fluvio-maritime.

Mots-clés: Erosion cotiére, Rechargement de plage, Flèche sableuse, Hydrodynamique, Morphodynamique, Saint-Louis 


\title{
Effectiveness of Recharging of the Pilot Bar Beach (Saint-Louis) After a Decade of Erosion
}

\author{
Omar Balde \\ Laboratoire de Sédimentologie et de Biostratigraphie, \\ Université Cheikh Anta Diop de Dakar, Sénégal \\ Dr. Issa Sakho
}

UMR Sciences Technologies Avancées et Développement Durable (UMR

STADD), Université Amadou Moctar Mbow de Dakar, Sénégal, Dakar, Sénégal. UMR-CNRS, Morpho dynamique continentale et côtière, Université de Rouen Normandie, Cedex France

Pr. Mouhamadoul Bachir Diouf

Laboratoire de Sédimentologie et de Biostratigraphie, Université Cheikh Anta Diop de Dakar, Sénégal

\section{Dr. Mamadou Bop}

Laboratoire Eau, Energie, Environnement et procédés industriels (LE3PI), Ecole Supérieure Polytechnique (ESP), Université Cheikh Anta Diop de Dakar, Sénégal

\section{Djibril Tine}

Laboratoire de Télédétection Appliquée de Dakar (LTA), Institut des

Sciences de la Terre, Université Cheikh Anta Diop de Dakar, Sénégal

\section{Wardou Ibouroi}

Laboratoire de Sédimentologie et de Biostratigraphie,

Université Cheikh Anta Diop de Dakar, Sénégal

\begin{abstract}
This work presents the results of a morpho-dynamic and sedimentological monitoring after beach nourishment and the consecutive formation of a lagoon. Topographic measurements have allowed determining the origin of the sediments present in the lagoon. The movement of the sediments is from the high beach to the lagoon. The analysis of current velocities shows a normal functioning of a fluvio-maritime system with high velocities during the flood period and low velocities during the ebb. Particle size analysis shows that fine-grained sediments are deposited in the underwater part of the beach profile. This first experiment in Senegal had positive results. The recharging of the Pilote Bar beach has favoured the formation of a lagoon. Sedimentation and increase in the height of the slopes of the neighboring beaches were also observed. The currents measured by the ADCP RIVER $1200 \mathrm{~kW}$, indicate a normal operation characteristic of a fluvio-
\end{abstract}


maritime zone.

Keywords: Coastal erosion, Beach nourishment, Sandy spit, Hydrodynamic, Moprhodynamic, Saint-Louis

\section{Introduction}

L'importance des études des côtes réside dans les conséquences géographiques et économiques des modifications du littoral (Mason, 1949). Les littoraux à travers le monde font actuellement l'objet d'aménagements continus aux conséquences parfois inattendues (Paskoff, 2007). Une action anthropique modifie presque toujours le fonctionnement naturel du littoral.

Les littoraux font l'objet de convoitises à cause des nombreux avantages qu'ils offrent: économie, pêche, tourisme, habitation etc. La population mondiale augmente et s'y concentre (Nicholls, 1998). Cependant, on assiste à une réduction de cet espace matérialisé par un recul du trait de côte induit par l'action des houles et/ou de la marée (Chaumillon et al., 2011; Faye, 2010).

L'évolution des côtes sableuses est dominée généralement par une tendance régressive (Paskoff, 2007). Ce phénomène est exacerbé par les effets du réchauffement climatique : hausse du niveau marin, augmentation de la fréquence des tempêtes (Bindoff et al., 2007; Solomon \& IPCC, 2007). En France, $24 \%$ du littoral est en érosion soit $1723 \mathrm{~km}$ de côtes sur un total de 7 124 km (Observatoire National sur les Effets du Réchauffement Climatique, 2015). Dans les régions tempérées comme au Québec, l'estuaire du Golf de Saint Laurent constitue un laboratoire pour comprendre les processus et les causes de l'érosion propre aux régions tempérées (Bernatchez \& Dubois, 2006). En Afrique de l'Ouest, l'évolution du trait de côte n'est pas régulière. Les zones d'érosion et d'accumulation se succèdent entre la Mauritanie et la Guinée (Faye, 2010). L'anthropisation accentue l'érosion par l'interruption des échanges dune-estran et aussi par l'artificialisation du trait de côte. Parallèlement, les ouvrages de protection, l'extraction de sédiment, l'occupation du sol des bassins versants accentuent le phénomène. Dans la région de Hi Phong au Vietnam, les aménagements autour du Port principal ont modifié l'équilibre littoral (Trinh et al., 2007).

Ainsi, face à cette situation, de nouvelles politiques de gestion moderne sont mises en place (Jeanson et al., 2011). Des techniques de protection comme l'enrochement sont remplacées par le rechargement artificiel de plage, une alternative pour les littoraux dont le rivage recule (Hamm et al., 2002; Harley et al., 2014; Newman, 1976). Au cours des 35 dernières années le rechargement artificiel a servi à la protection des côtes en Caroline du nord (Rogers, 2000). En Languedoc- Roussillon, ces pratiques de rechargement sont réalisées de façon ponctuelle à des échelles locales en se 
servant des sédiments recueillis par dragage ou des zones d'accrétion (Bâ et al., 2007).

Toutefois, la conception de cette méthode dépend des objectifs fixés. A cet effet, les sédiments ajoutés au système peuvent aider à dissiper l'énergie des vagues pendant les tempêtes (Harley et al., 2014). Techniquement un bon rechargement utilise des sédiments de même granulométrie, voire légèrement plus grossier (Newman, 1976).

A Saint Louis, une brèche ouverte sur la flèche sableuse de la Langue de Barbarie, au Nord du Sénégal, a eu des conséquences désastreuses sur le bief fluvial en aval. Le creusement du canal devrait permettre l'écoulement rapide des eaux d'inondation qui ont fortement menacé la ville de Saint-Louis pendant les crues de 2003. Cette brèche sur la Langue de sable séparant le fleuve Sénégal de l'Océan Atlantique n'est pas une première puisque la flèche de Saint Louis a connu de nombreuses ruptures naturelles au cours de son évolution (Gac et al., 1982). A partir de son extremité, l'accroissement de la langue de Barbarie s'allongeait régulièrement vers le Sud sous l'effet de la dérive littorale et la rendait instable provoquant une rupture de la flèche et l'installation d'une nouvelle embouchure (Gac et al., 1982).

L'environnement morphodynamique du delta évoluait considérablement mais le système se reconstituait progressivement, la partie distale de l'ancienne flèche évoluait en lagune et la nouvelle flèche, écourtée, reprenait son allongement vers le Sud. Le creusement du canal d'évacuation des eaux pluviales en 2003 pour éviter l'inondation de la ville de St Louis a logiquement généré une modification du système mais celui-ci ne s'est pas régénéré de lui-même. La brèche s'est élargie considérablement et la rive gauche du fleuve s'est retrouvée soudainement exposée à l'action de la houle dont elle était protégée par la flèche. Une des conséquences de cette évolution est le recul de la ligne de rivage dans le secteur (Bâ et al., 2007; Faye, 2010). Plus au Sud, d'autres formes d'évolutions régressives font l'objet de menaces sérieuses contre des territoires plus étendus et des écosystèmes très variés.

Les risques d'inondation évités à Saint Louis, il fallut désormais s'attaquer aux nouveaux risques engendrés par cette ouverture. En effet, les villages exposés à la dynamique marine sont confrontés à des risques d'érosion et de submersion marine. Doun Baba Dieye, Pilote Bar et Tassinére, villages situés au Sud de la brèche, subissent successivement l'action dévastatrice des vagues. Cette situation a provoqué le déplacement des populations et la salinisation des terres. L'Etat du Sénégal et la commune de Ndieben Gandiole décident, après des études, de protéger le village de Pilote Bar par un rechargement artificiel de sa plage.

Cette première à Pilote Bar est donc expérimentale d'où la nécessite d'étudier l'efficacité et l'impact du rechargement sur l'environnement fluviomarin de Pilote Bar. Cet article cherchera à mesurer l'impact de ce 
rechargement. Pour y parvenir trois principaux facteurs vont être analysés : l'évolution de la géomorphologie, les processus sédimentaires et enfin l'hydrodynamisme du milieu.

\section{Matériels et Méthodes}

\subsection{Présentation de la Zone d'étude}

La zone d'étude se situe au Nord du Sénégal à l'embouchure du fleuve Sénégal. Elle est comprise entre les latitudes $16^{\circ} 23^{\prime}$ et $16^{\circ} 35^{\prime} \mathrm{N}$ et les longitudes $15^{\circ} 45^{\prime}$ et $16^{\circ} 15^{\prime} \mathrm{O}$. Nous retrouvons dans cette partie la flèche sableuse de la Langue de Barbarie et le chenal fluvial. Cette flèche sableuse, allongée vers le Nord sépare l'Océan atlantique à l'Ouest et le fleuve Sénégal à l'Est (Fig.1).

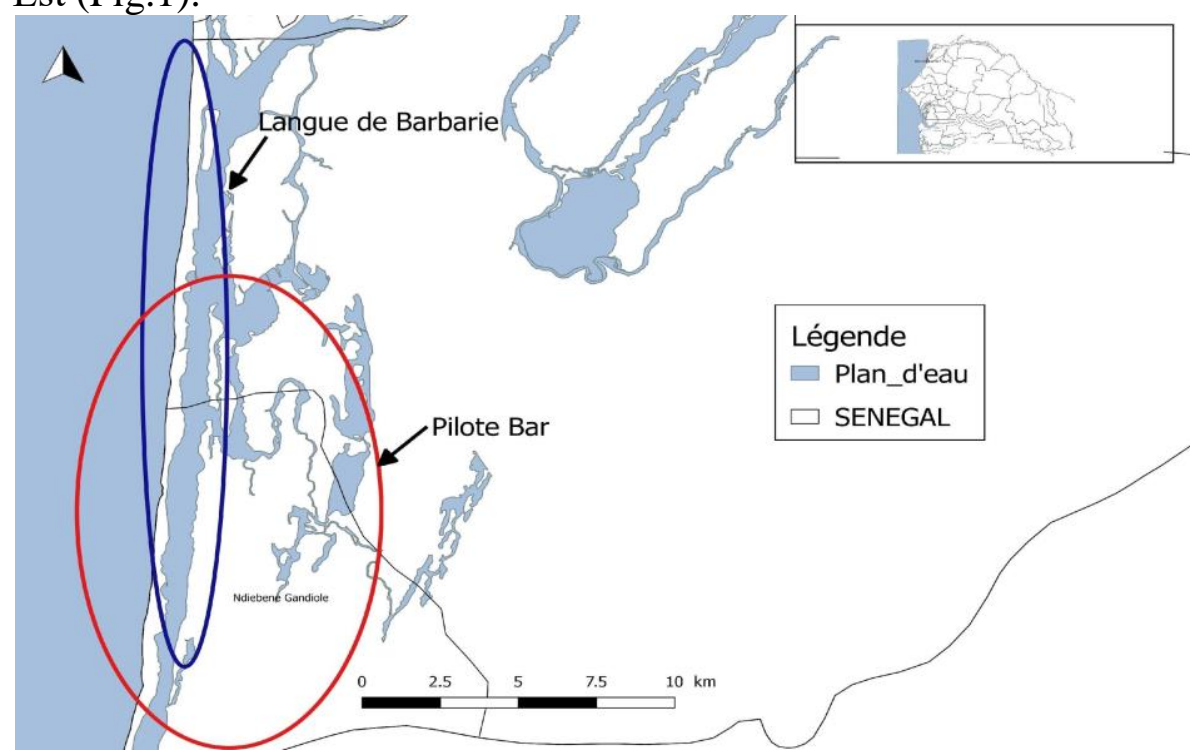

Figure 1. Localisation de la zone d'étude

\subsection{Stratégie de l'étude}

La stratégie repose sur un suivi morphodynamique, sédimentologique et courantologique du segment littoral de Pilot Bar. Plusieurs missions de terrain ont été organisées annuellement de décembre 2016 à nos jours. Un DGPS (Differential Gobal Positioning System) a permis de suivre l'évolution morphologique des différentes unités de la plage.

L'étude hydrodynamique est realisée par analyse des données marégraphiques. Un ADCP (Acoustic Doppler Current Profiler) parcourt un profil long shore et un profil cross shore toutes les 30 minutes au cours d'un cycle de marée. Les données sur la hauteur de la marée sont directement enregistrées et le post-traitement est fait par le logiciel Win river 2. 


\subsection{Caractéristiques de l'Ouvrage}

L'option protection par engraissement de la plage est la solution choisie par les autorités pour compenser l'érosion de la plage consécutive à l'ouverture et à l'évolution régressive de la brèche (Fig. 2). Pour être efficace, l'ouvrage a été dimensionné pour respecter les caractéristiques géométriques et granulométriques suivantes :

- Largeur à la crête de l'engraissement: 20 mètres

- Longueur de la côte à protéger : sept cent mètres linéaires $(700 \mathrm{ml})$

- Hauteur moyenne du sable à mettre en place : $2 \mathrm{~m}$

- Pente de talus : $1 \mathrm{v}: 5 \mathrm{~h}\left(11^{\circ} 5\right)$

- Matériaux à utiliser : sable de dune

Les matériaux apportés doivent être identiques à ceux sur place.

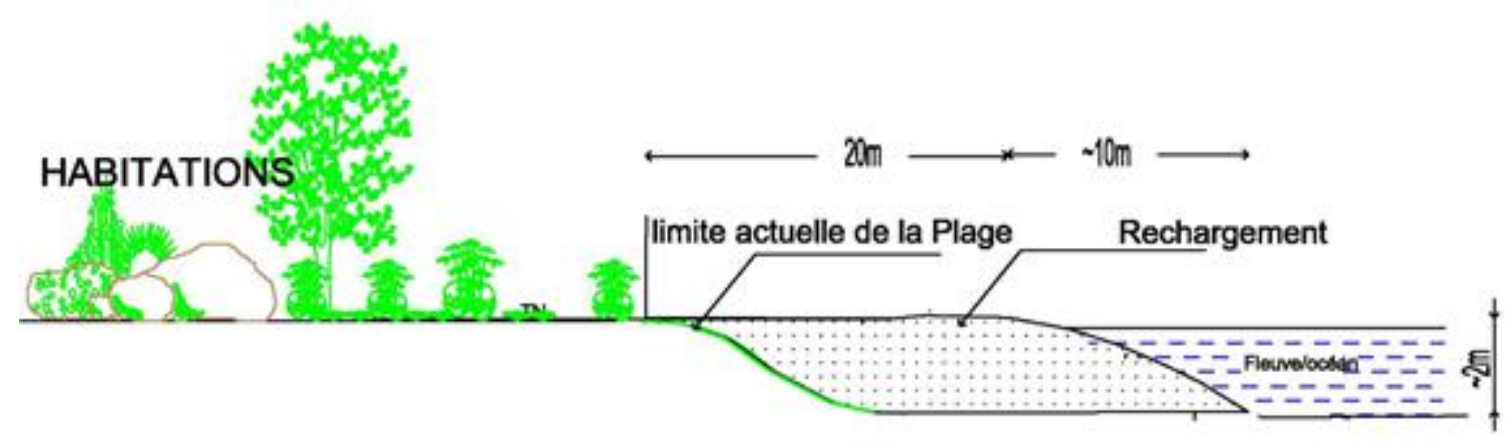

Figure 2. Schéma de l'engraissement de la plage de Pilote Bar (source : DEEC, 2015)

Pour la réalisation de l'ouvrage, le sable doit correspondre aux caractéristiques granulométriques de la plage de Pilote bar c'est-à-dire $75 \%$ de sable fins (DEEC, 2015). La quantité de matériaux nécessaire est estimée à $46600 \mathrm{~m}^{3}$ environ, il s'agit de volume mesuré après compactage (Photo 1). 


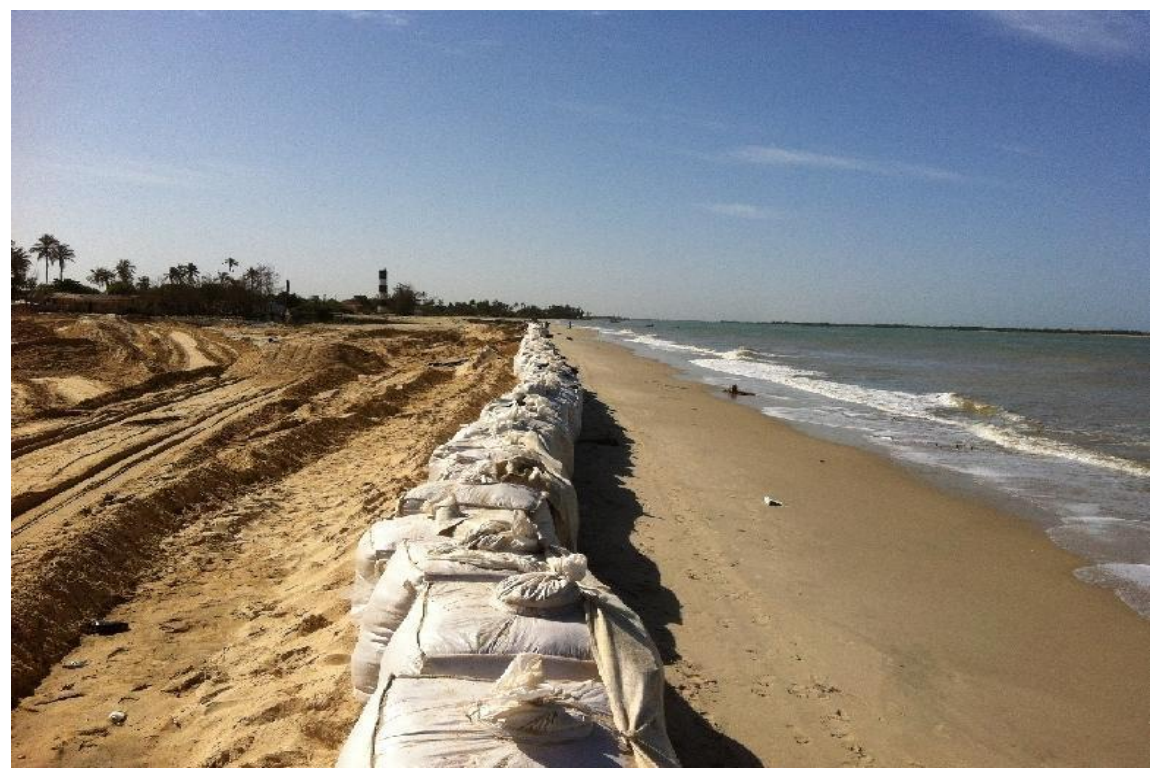

Photo 1. Travaux de rechargement de la plage de Gandiol en 2015 (source : DEEC, 2015)

\subsection{Méthode d'acquisition des données topographiques}

La méthodologie s'appuie sur une comparaison de données de topographie acquises sur le terrain avec un DGPS (Photo 2). Ces données vont permettre d'étudier la géomorphologie mais aussi de calculer le bilan sédimentaire.

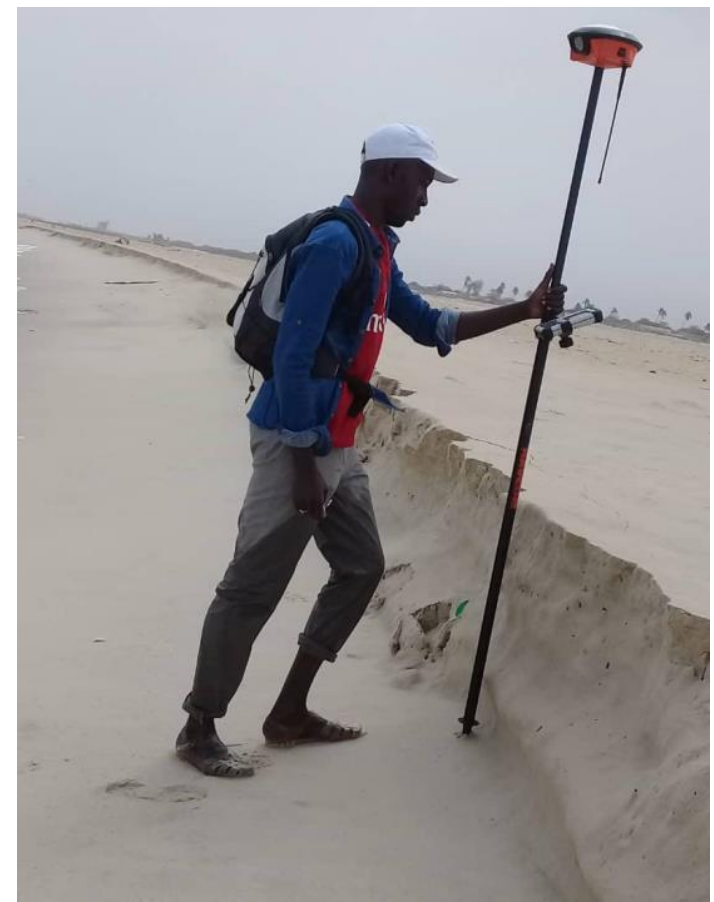

Photo 2. Mesure topographique au DGPS 
Le principe de fonctionnement du DGPS repose sur l'emploi d'une "base" (ou Pivot) GPS positionné sur un point (borne géodésique à RAO dans le cadre de cette étude) connu très précisément, on calcule en temps réel les écarts entre le point connu et la position calculée par les observations satellites puis on transmet ces valeurs de correction vers un "mobile GPS" (ou Rover"). En moyenne plus de 700 points sont levés par mission. Ils couvrent la plage de pilote Bar et la flèche en formation. Les données sont ensuite calculées et corrigées pour avoir des résultats avec des précisions millimétriques. Les modèles numériques obtenus ont été réalisés avec le logiciel Global Mapper. Les mesures de profil de plage effectuées à l'aide d'une station totale, ont permis de suivre l'évolution de la plage, d'identifier les zones en érosion et celles en accrétion (Photo 3).

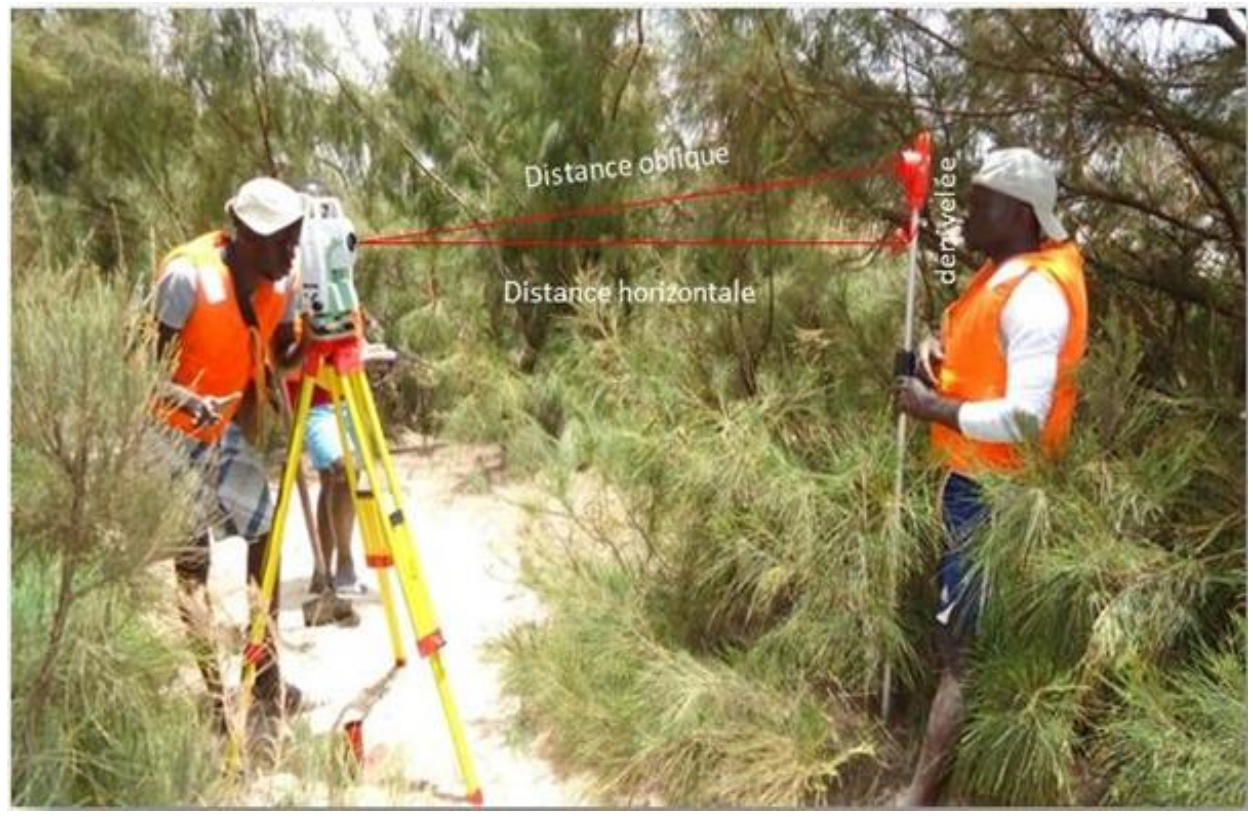

Photo 3. Mesure topographique avec la station totale

La comparaison de deux profils (Fig. 3) sur un même transect permet d'estimer le bilan sédimentaire. Un bilan négatif est donc un signe d'érosion. 


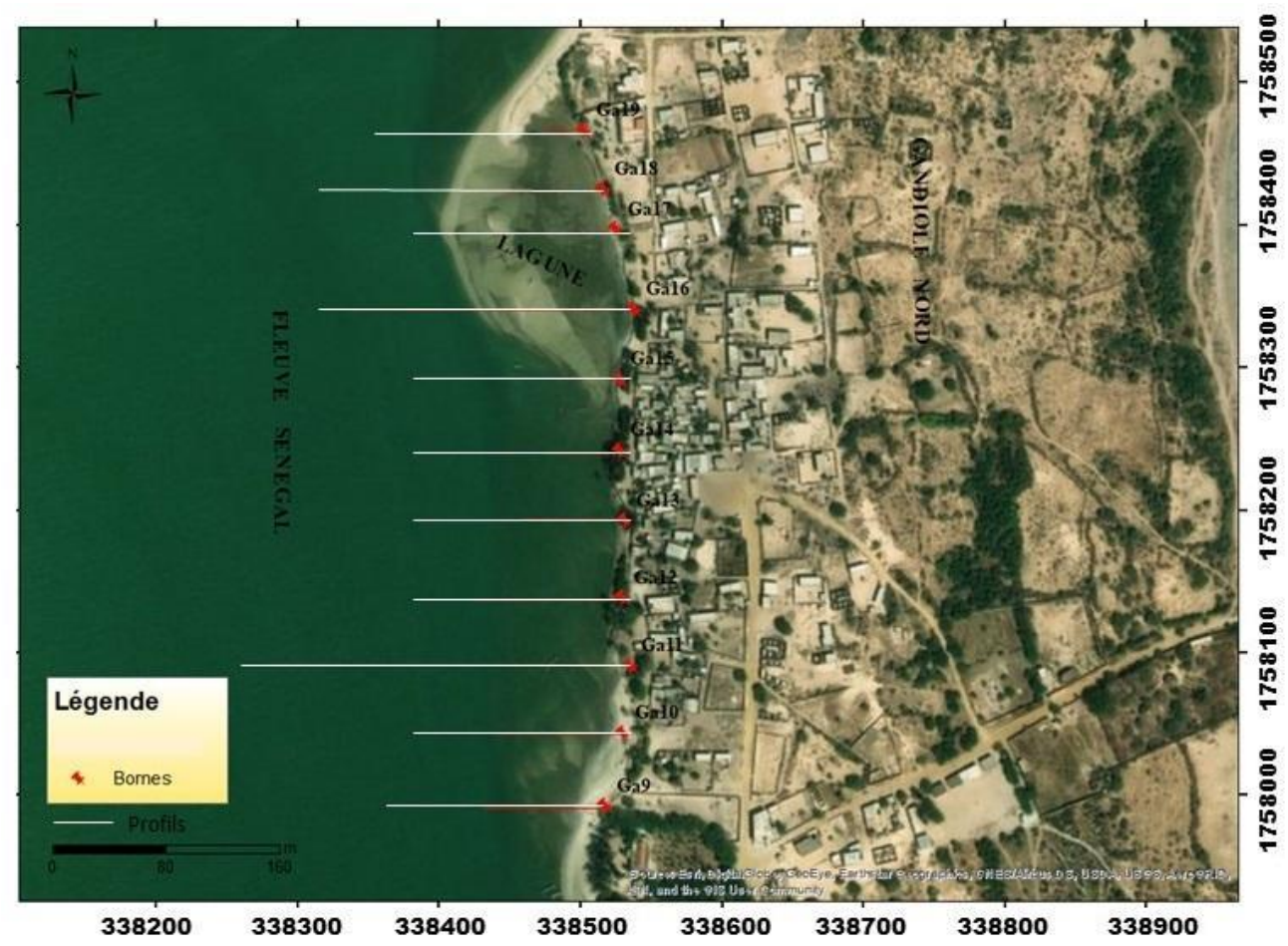

Figure 3. Localisation des profils topographiques

L'appareil est placé sur une borne repère fixe et orienté perpendiculairement à la ligne de rivage à partir d'un repère arrière fixe. Les points sont pris à chaque changement de pente par le porteur de la canne tout au long du profil jusqu'à la plage sous-marine.

\subsection{Mesures des hauteurs d'eau et des vitesses de courants de marée}

L'étude hydrodynamique est faite à l'aide des données d'un « Acoustic Doppler current profiler » $(A D C P)$. Cet outil permet de mesurer la vitesse du courant le long d'un profil donné. Pour cette étude deux profils ont été effectués : un profil parallèle au rivage passant devant la passe et un profil perpendiculaire au rivage qui a permis d'étudier les courants à l'intérieur de la lagune (Fig.4). 


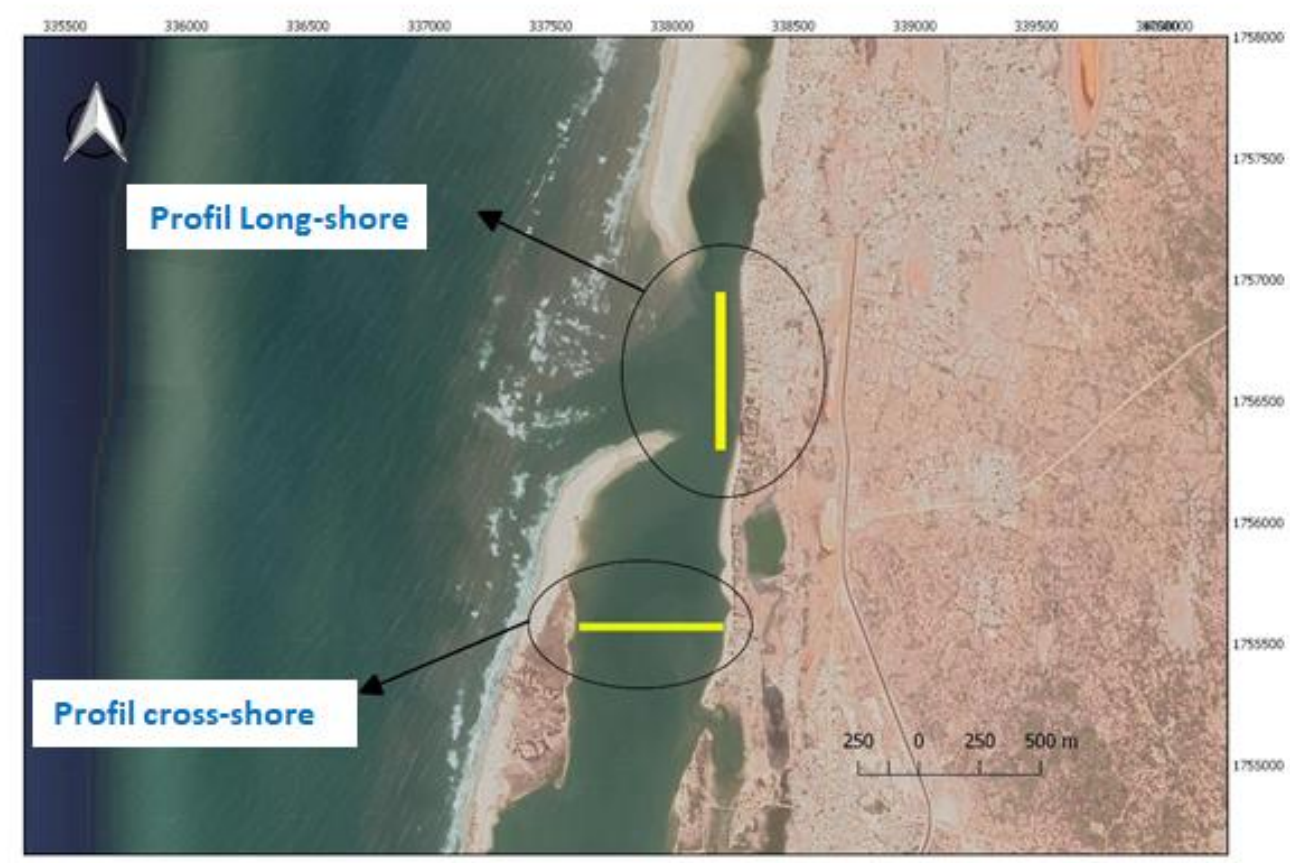

Figure 4. Localisation des transects de mesure des vitesses de courant

Un Profileur acoustique mobile $A D C P$ doppler a servi à la réalisation de l'étude hydrographique (Photo 4). Ce dispositif multi faisceaux donne la vitesse et la direction des courants qui traversent le profil. Les profils de vitesses de courant sont acquis toutes les $30 \mathrm{mn}$, de $9 \mathrm{~h} 00$ à $19 \mathrm{~h} 00$. Le trajet défini pour les mesures de courant est situé devant la passe de la lagune (Fig.4). La courantométrie a été réalisée en juillet 2017 et en décembre 2019. La première opération a été réalisée en période de Morte-eau avec un coefficient de marée de 59. En décembre, par contre, le coefficient de marée a été de 104 (très haut). Les données sont ensuite post traitées par le logiciel Win river 2. 


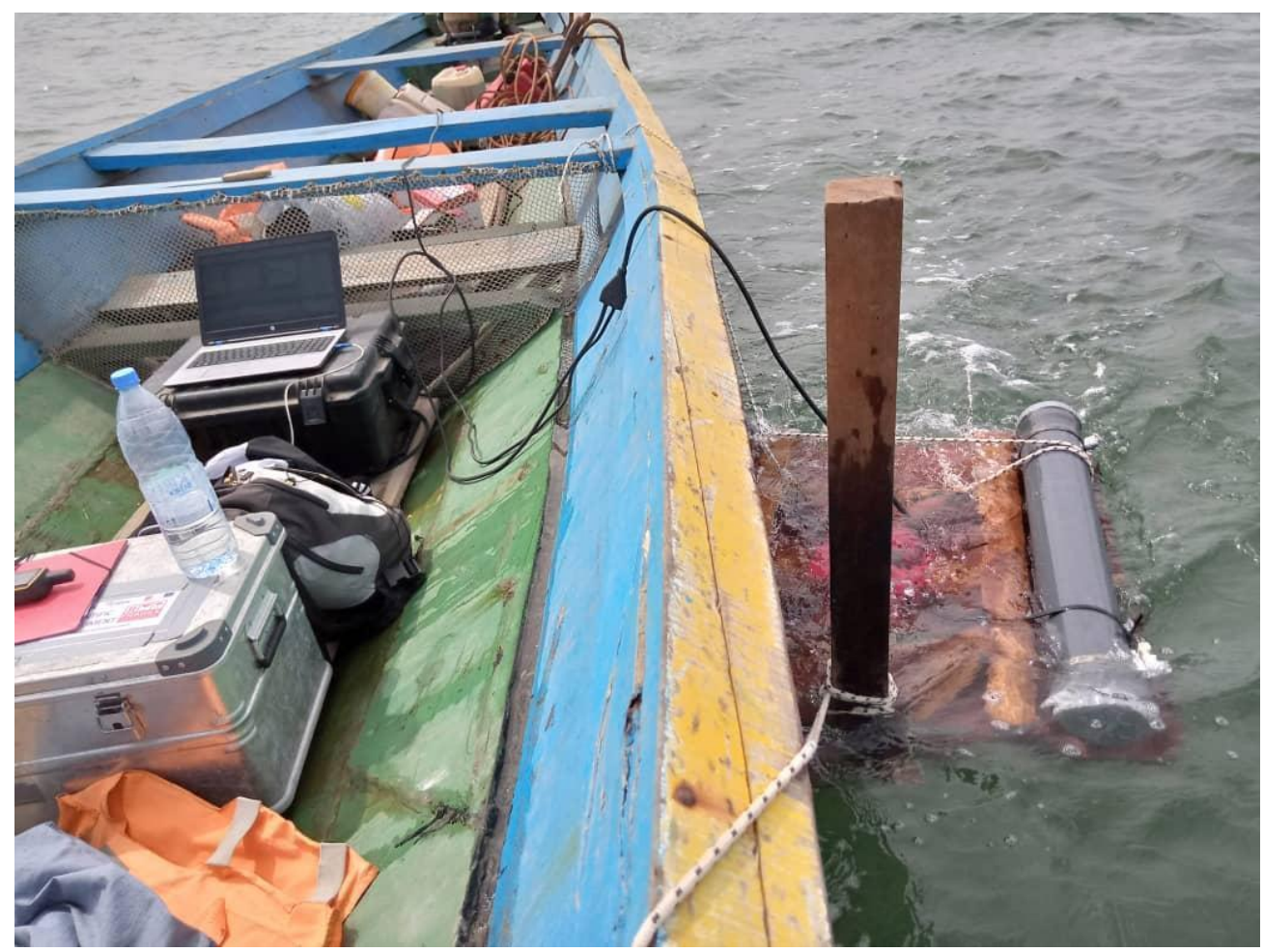

Photo 4. Dispositif de Mesure de vitesse Courant avec ADCP

Le dispositif comprend un ordinateur portable relié à l'ADCP, une batterie de recharge et un flotteur qui maintient l'ADCP en surface. Tout ce dispositif est embarqué dans une pirogue qui effectue les rotations entre les point A et B du profil. Deux trajets ont été définis : cross-shore (environ 400 $\mathrm{m}$ ) et long -shore (environ $600 \mathrm{~m}$ ) au niveau de la lagune.

\subsection{Prélèvements sédimentaires}

Pour l'étude sédimentologique, les prélèvements sont faits sur les unités morphologiques traversées par les profils topographiques (Fig.5). Les échantillons sont ensuite traités au laboratoire de sédimentologie. La granulométrie des échantillons est déterminée par tamisage. 


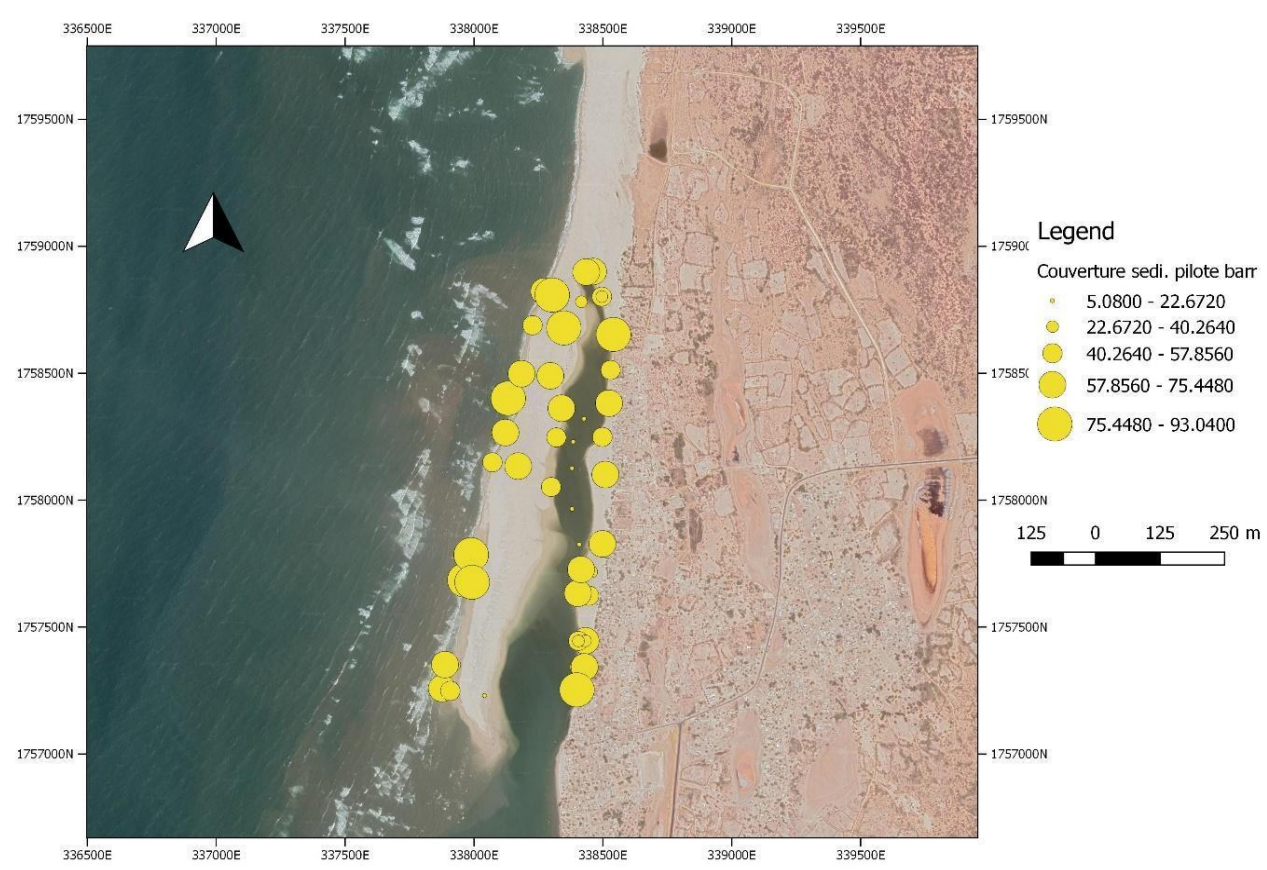

Figure 5. Cartographie des points d'échantillonnage de sédiment

L'analyse des données s'est faite à partir des «indices granulométriques » calculés selon la méthode de Folk et Ward (1957). Ces indices indiquent le mode de dépôt qui, à son tour, permet de caractériser l'environnement de dépôt des sédiments. Le plus utilisé parmi eux est l'indice d'asymétrie (Sk-Skewness) (Equation 1).

$$
\text { Equation 1: } \quad S K=\frac{\varphi_{84}+\varphi_{16}-2 \varphi_{50}}{2\left(\varphi_{84}-\varphi_{16}\right)}+\frac{\varphi_{95}+\varphi_{5}-2 \varphi_{50}}{2\left(\varphi_{95}+\varphi_{50}\right)}
$$

La Moyenne et la Médiane reflètent la distribution granulométrique des sédiments. Elles sont de bons indicateurs de l'énergie du milieu pendant le dépôt des sédiments. La Médiane correspond à $50 \%$ des grains sur la courbe cumulative. La Moyenne (Grain moyen) est calculée à partir de l'équation 2.

Equation 2: $\quad M=\frac{\varphi_{16}+\varphi_{50}+\varphi_{84}}{3}$

Les paramètres de position sont convertis en unité phi avant le calcul des indices selon l'équation 3.

Equation 3:

$$
\begin{gathered}
\text { phi } \emptyset=\ln d / \ln 2 \\
\mathrm{~d}=\text { diamètre grain en } \mathrm{mm}
\end{gathered}
$$




\section{Résultats}

Dans le cadre du suivi de l'ouvrage, l'étude a démarré en décembre 2016. Les données topographiques recueillies confirment les signes d'érosion déjà notés dans le cadre du suivi régulier initié par l'équipe de Géologie marine de l'Université Cheikh Anta DIOP de Dakar depuis plus d'une décennie à partir de l'Observatoire Dynamique des Côtes de Gandiole. Le secteur Nord de la plage de Pilote Bar montrait des signes d'érosion et un début de formation de banc de sable .

\subsection{Evolution du profil de plage entre 2015 et 2017}

Le suivi morphologique avec une station totale Leica avait mis en exergue un début de sédimentation au Nord du phare correspondant à la partie proximale (Fig. 6) de la flèche en formation.

\section{Profil 18}

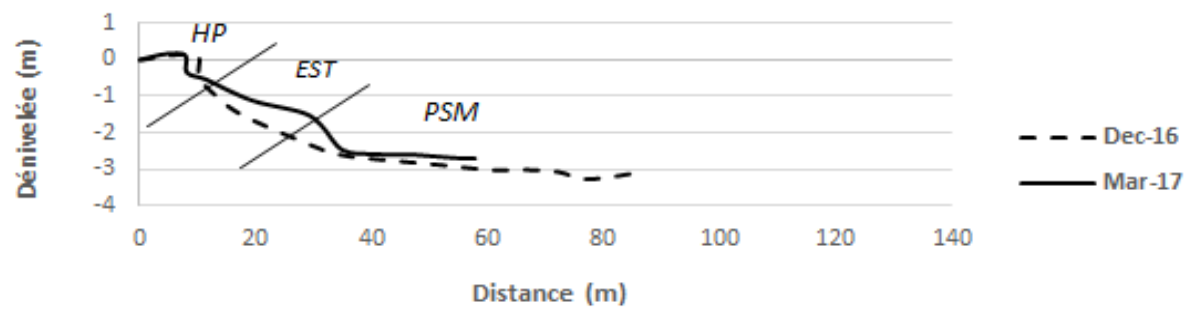

\section{Profil 14}

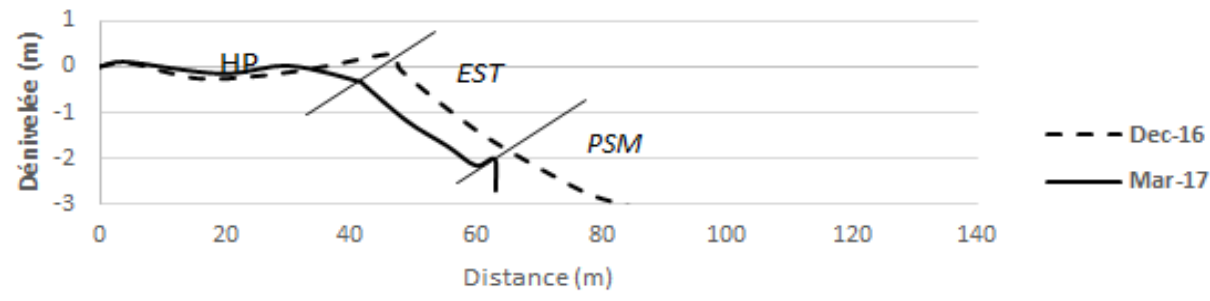

\section{Profil 10}

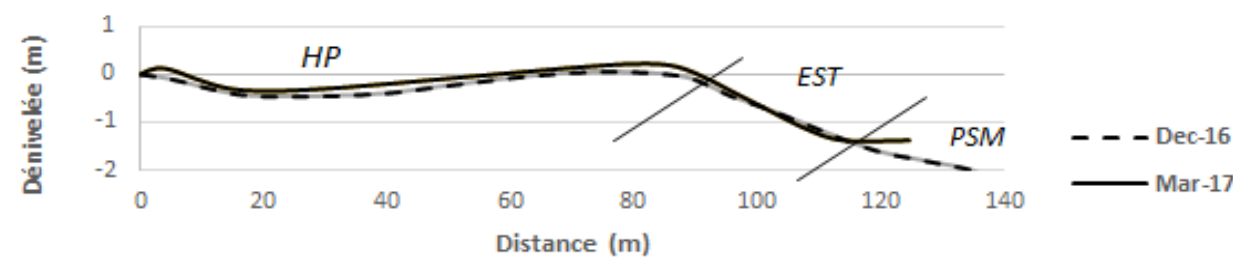

Haute plage $=H P \quad$ Estran $=E S T$ Plage sous marine $=P S M$

Figure 6. Evolution des profils de plage Gandiol entre 2016 et 2017 
Le profil 14 situé au centre de la plage montre une plage concavoconvexe, haute plage et estran bien distincts. Les pentes sont faibles vers la haute plage $(0,77$ et $1,68 \%)$ et fortes sur l'estran $(11,64$ et $10,34 \%)$. On peut noter une accrétion sur la haute plage et une forte érosion sur l'estran et la plage sous- marine. L'érosion reste dominante le long du profil.

Le profil 10 mesuré entre décembre 2016 et mars 2017 présente une forme concavo-convexe, haute plage et estran bien distincts. Les pentes sont faibles sur la haute plage environ $(0,25$ à $3 \%)$; modérées et fortes sur l'estran $(4,60$ et $6,47 \%)$. La plage présente une accrétion au niveau de la haute plage et l'estran, suivi d'un léger équilibre et puis une faible érosion vers la plage sous-marine.

\subsection{Evolution morphosédimentaire saisonnière}

Le modèle numérique de terrain indique un allongement de la petite flèche entre février et décembre 2019 (Fig. 7). La fusion des bancs de sable semble donner naissance à une flèche, isolant une lagune sur la rive gauche du chenal. Les sédiments du rechargement après quelques mois se retirent vers le large pour former des bancs de sable.

Le modèle met en exergue plusieurs bancs de sable séparés par de petites passes (Fig. 7). Ces bancs estuariens se forment au niveau des zones où le transport sédimentaire résiduel est convergent entre les chenaux respectivement dominés par le flot et le jusant (Bertin \& Chaumillon, 2005). Situés à des altitudes faibles $(-2 \mathrm{~m})$, ils sont caractérisés par des dépôts récents de sédiments fins ce qui donne un caractère fragile et temporaire à ces dépôts. 

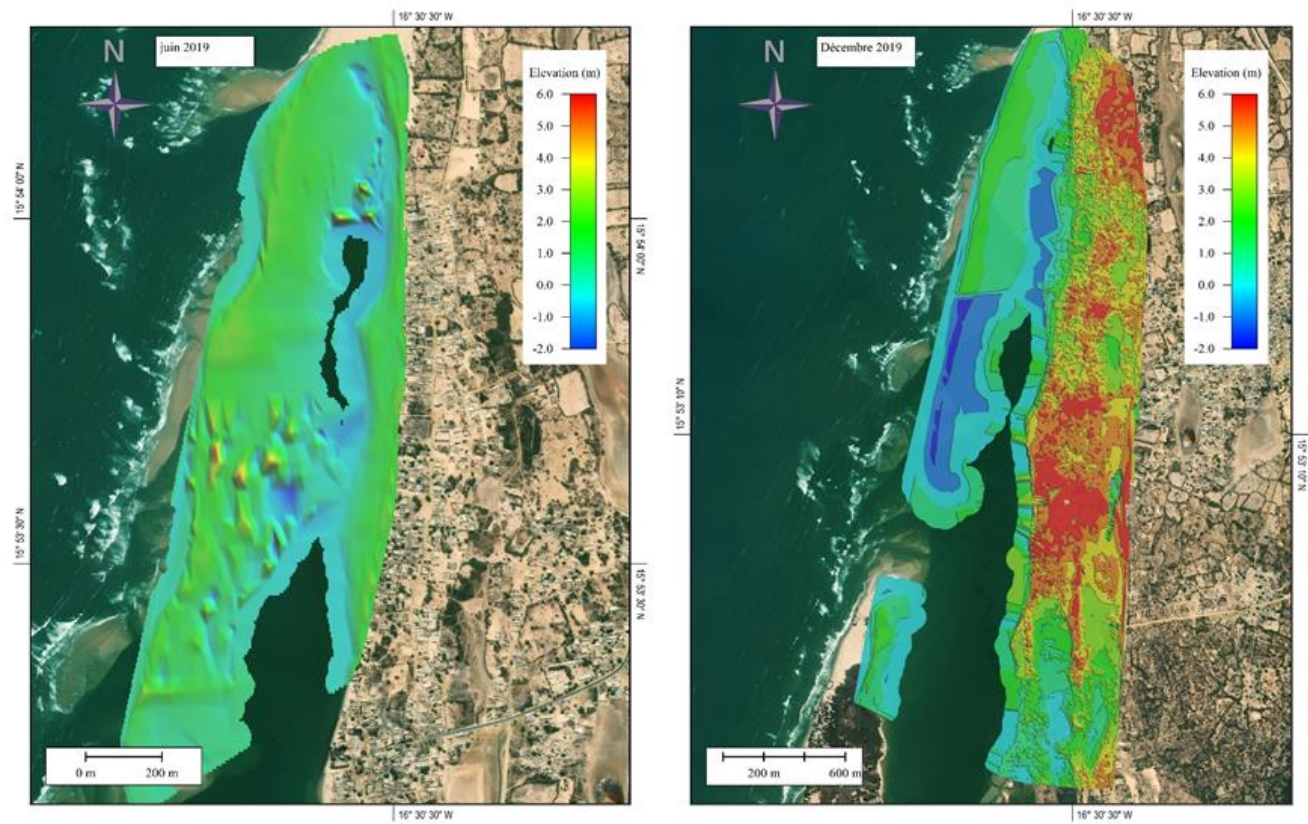

Figure 7. Modèle numérique de terrain de la plage de Pilote Bar. (A) Aout 2019, (B) Décembre 2019

\subsection{Evolution des courbes granulométriques}

Les échantillons de sable prélevés à Pilote Bar sont essentiellement composés de sables moyens à grossiers à l'exception des échantillons de la Lagune qui sont fins (Fig. 8).

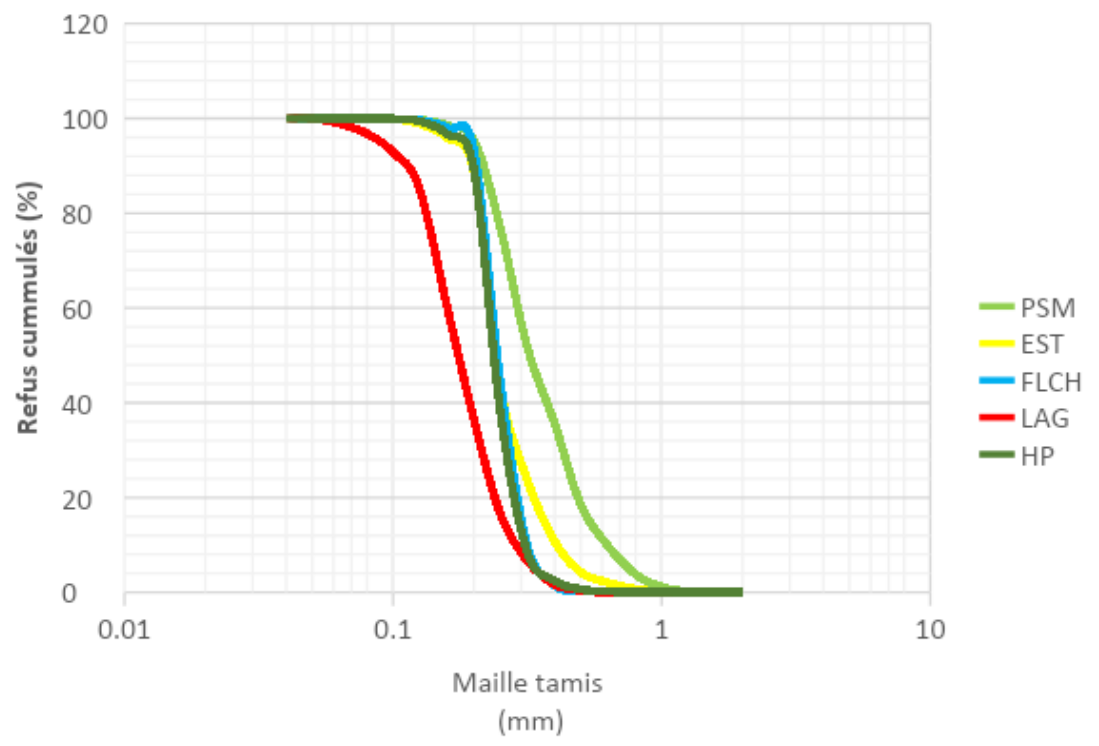

Figure 8. Courbes granulométriques des sédiments de la Lagune de Gandiole (PSM : Plage sous-marine, EST : Estran, FLCH : Flèche, LAG : Lagune, HP : Haute plage) 
L'étude granulométrique réalisée au préalable à Gandiole, montrait la présence d'éléments grossiers dans la partie sous-marine du profil de plage $(0,219$ à $0,378 \mathrm{~mm})$ et d'éléments fins dans la partie haute de la plage. Ce changement est dû au rallongement de la flèche qui réduit l'action de la dynamique marine dans la lagune favorisant le dépôt d'éléments fins.

Les données sédimentologiques récentes montrent un sable fin environ 2 phi dans la lagune et du sable moyen à grossier 1,5 phi de part et d'autre de celle-ci. Les sédiments sont bien classés et présentent une asymétrie négative synonyme d'un dépôt fait par un courant fort (tableau 1).

Le tableau 1 renferment les valeurs en unité phi des indices granulométriques.

Tableau 1. Indices granulométriques

\begin{tabular}{|c|c|c|c|c|c|c|}
\hline $\begin{array}{c}\text { Les unités } \\
\text { morphologiques }\end{array}$ & $\begin{array}{l}\text { Grain } \\
\text { Moyen } \\
(\mathrm{Mz})\end{array}$ & $\begin{array}{c}\text { La } \\
\text { Médiane }\end{array}$ & $\begin{array}{l}\text { Indice de } \\
\text { Classement } \\
\text { ou de triage }\end{array}$ & $\begin{array}{c}\text { Indice } \\
\text { d'asymétrie } \\
\text { ou Skewness }\end{array}$ & $\begin{array}{c}\text { Indice } \\
\text { d'Acuité } \\
\text { ou } \\
\text { Kurtosis }\end{array}$ & Interprétation \\
\hline $\begin{array}{l}\text { Plage sous- } \\
\text { marine }\end{array}$ & 1,54 & 1,59 & 0,621 & $-0,189$ & 1,000 & $\begin{array}{l}\text { Sable moyen, } \\
\text { Moyennement à } \\
\text { bien classé, } \\
\text { courant de } \\
\text { dépôt fort }\end{array}$ \\
\hline Lagune & 2,47 & 2,50 & 0,472 & $-0,197$ & 0,814 & $\begin{array}{l}\text { Sable fin, bien } \\
\text { classé, courant } \\
\text { de dépôt fort }\end{array}$ \\
\hline Estran & 1,93 & 2,07 & 0,459 & $-0,409$ & 1,152 & $\begin{array}{l}\text { Sable moyen, } \\
\text { bien classé }\end{array}$ \\
\hline Haute plage & 2,04 & 2,10 & 0,311 & $-0,244$ & 1,219 & $\begin{array}{l}\text { Sable fin, Très } \\
\text { bien classé, } \\
\text { courant de } \\
\text { dépôt fort }\end{array}$ \\
\hline Flèche & 2,01 & 2,05 & 0,262 & $-0,237$ & 1,013 & $\begin{array}{l}\text { Sable fin, Très } \\
\text { bien classé, } \\
\text { courant de } \\
\text { dépôt fort }\end{array}$ \\
\hline
\end{tabular}

\subsection{Caractéristiques des conditions hydrodynamiques à}

\section{l'embouchure de la Lagune de Pilote Bar}

L'étude a été menée sur le bief maritime de Pilote Bar après le rechargement de sa plage.

\subsubsection{Le profil long-shore de Pilote Bar}

La courantométrie a été réalisée le 24 Décembre 2019. Cette date correspond à une période de vives eaux avec un coefficient de marée de 86 . Les profils de marée semi-diurne de la journée du 24 décembre sont présentés sur les figures 9 et 10. La figure 9 indique des vitesses de courant faibles pendant la période de jusant avec une vitesse moyenne de $0,183 \mathrm{~m} / \mathrm{s}$. 

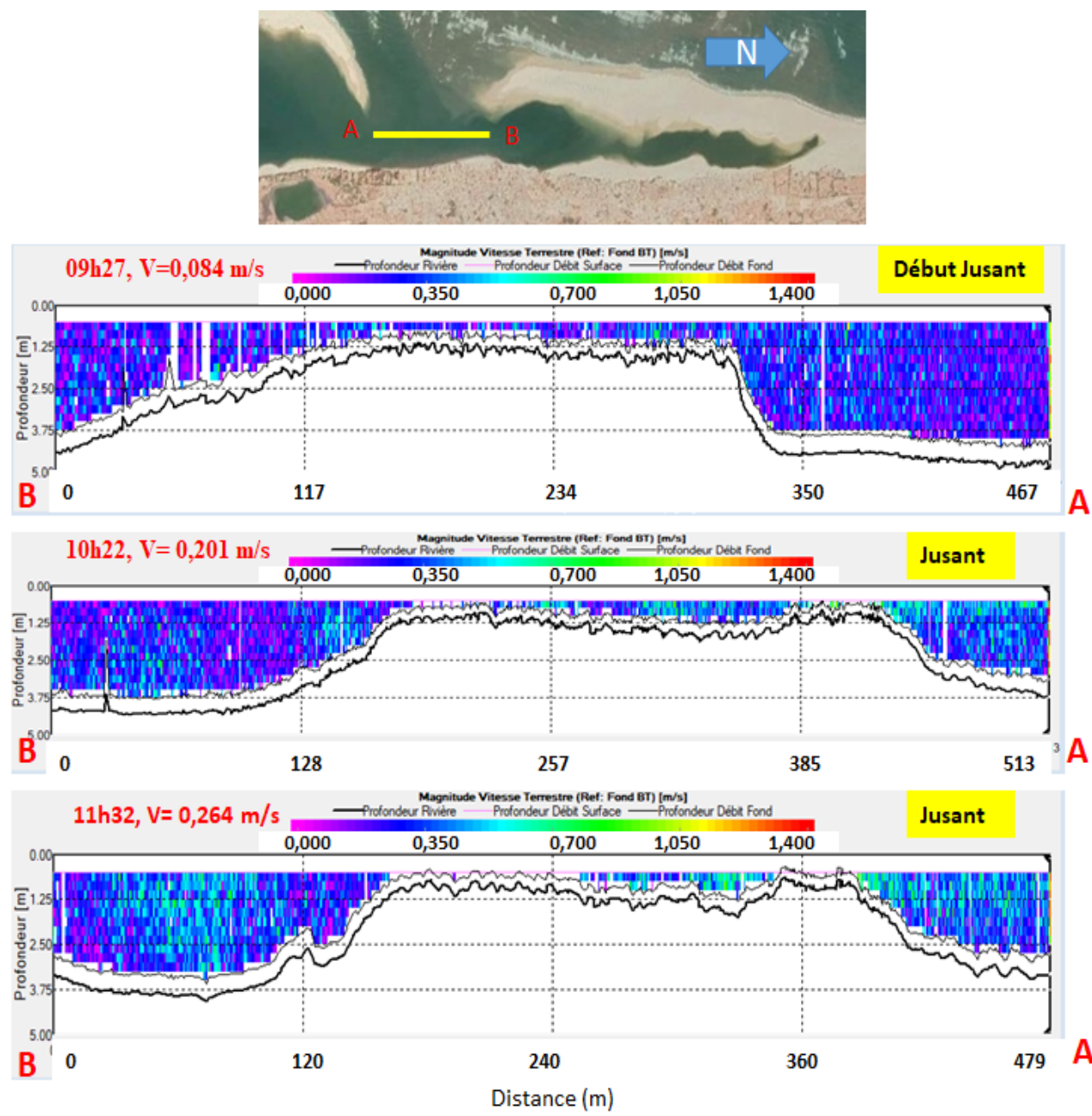

Figure 9. Profils de vitesse de courant (profil long-shore) durant une période de Jusant

La figure 10 indique des vitesses moyennes de courant plus élevées $(0,462 \mathrm{~m} / \mathrm{s}$ en moyenne). Ces courants de flot sont plus forts au sud du haut fond.

Les profils montrent la présence d'un banc de sable aux environs de $200 \mathrm{~m}$ en face de la passe. Les courants sont plus forts au sud de ce haut fond en couleur verte et jaune (Fig. 10). Les ensembles vides obtenus après post traitement indiquent la forte agitation du milieu pendant les mesures de vitesses. 


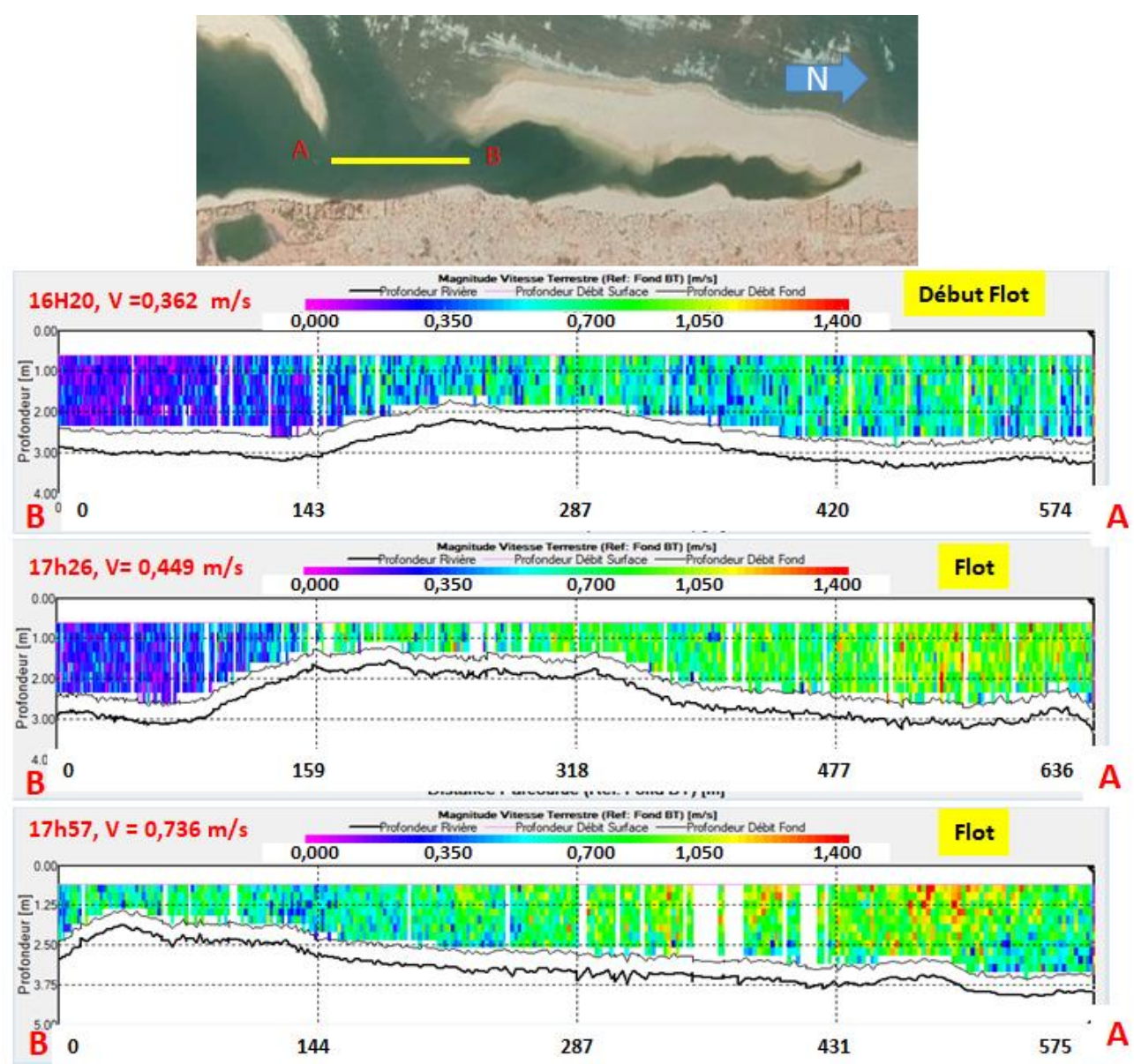

Figure 10. Profils de vitesse de courant (profil long-shore) durant une période de courant de flot

Les résultats montrent globalement une marée diurne avec deux types de courant :

- des courants de jusant faibles de $0,183 \mathrm{~m} / \mathrm{s}$, déplaçant un débit total d'eau de $71,64 \mathrm{~m}^{3} / \mathrm{s}$.

- des courants de flot forts de $0,462 \mathrm{~m} / \mathrm{s}$ déplaçant un débit total d'eau de $86,50 \mathrm{~m}^{3} / \mathrm{s}$.

\subsubsection{Le profil cross-shore de la lagune}

La courantométrie a été réalisée le 11 Février 2020. Cette date correspond à une période de vives eaux avec un coefficient de marée de 101. Le profil se trouve à environ $1 \mathrm{~km}$ au sud de la brèche et relie les deux rives du chenal fluvial.

Les vitesses moyennes de courant sur le profil cross-shore sont faibles $(0,311 \mathrm{~m} / \mathrm{s}$ en moyenne). La marée diurne du 11 février est représentée sur les 
figures 11 et 12 avec, notamment des vitesses moyennes de courant de flot fort (Fig. 11) et celles de jusant faible (Fig. 12).
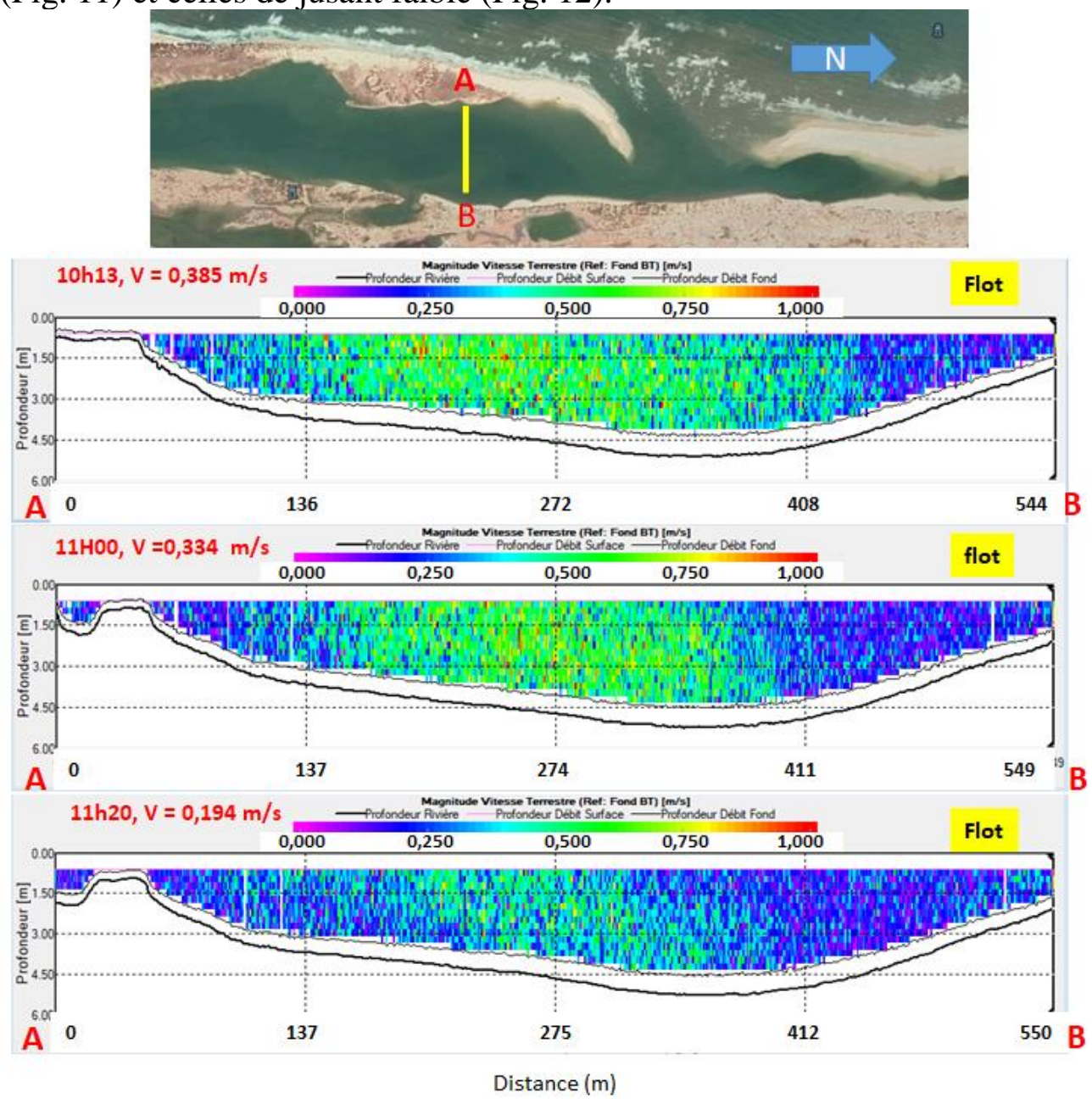

Figure 11. Profils de vitesse de courant (profil cross-shore) durant une période de courant de flot 

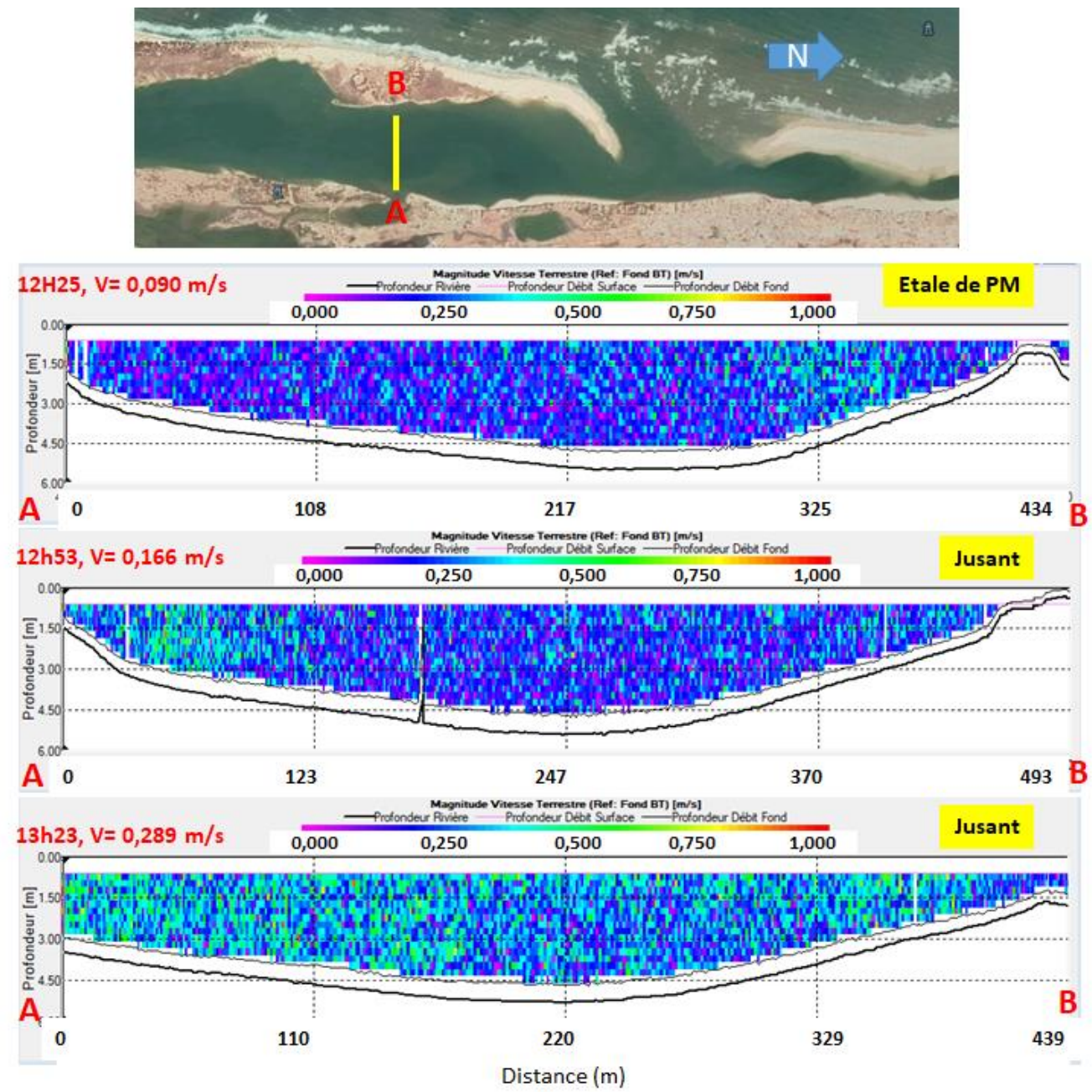

Figure 12. Profils de vitesse de courant (profil cross-shore) durant une période de courant de jusant

En résumé, le profil cross-shore indique deux principaux courants dans la journée du 11 février :

- des courants de flot forts de $0,311 \mathrm{~m} / \mathrm{s}$, déplaçant un débit total de $497,50 \mathrm{~m}^{3} / \mathrm{s}$ d'eau.

- des courants de jusant faibles de $0,167 \mathrm{~m} / \mathrm{s}$, déplaçant un débit total de $233,58 \mathrm{~m}^{3} / \mathrm{s}$ d'eau.

\section{Discussion}

Du risque d'inondation fluviale au risque de submersion marine : la brèche de Saint Louis

En Octobre 2003, la crue exceptionnelle sur le fleuve et l'ouverture complète des vannes du barage de Diama, ont entraîné une inondation sur la 
zone basse de Saint Louis. Cette montée rapide et soudaine du plan d'eau du fleuve (1,94 m dans la nuit du 3 au 4 octobre dépassant son seuil d'alerte de $1,74 \mathrm{~m}$ ) constituait une menace pour la ville de Saint Louis.

En definitive, l'ouverture de la brèche à $7 \mathrm{~km}$ au sud de Saint-Louis, a permis de maintenir le niveau en dessous de $100 \mathrm{~cm}$ et enrayer les risques d'inondation liés au débordement du chenal fluvial (Durand et al., 2010; B. A Sy, s. d.).

Les risques liés à l'inondation ont été favorisés par un retour de la pluviométrie entre $2002-2011$. A cela s'ajoutent les problèmes d'occupation du sol à Saint Louis, particulièrement au niveau de la Langue de Barbarie. En conclusion, cet aménagement a permis de maintenir le niveau du fleuve en dessous de la côte d'alerte.

L'élargissement du canal constitue un risque de submersion marine. Sa longueur évolue à une vitesse d'environ $500 \mathrm{~m} / \mathrm{an}$ (Bâ et al., 2007). La brèche qui devait permettre de réduire les surfaces inondées aux alentours de Saint Louis, a engendré de nouveaux risques côtiers dans la région. L'action des vagues a fini par submerger et entrainer le village de Doun Baba Dieye sous les eaux.

\section{Efficacité du chargement de la plage}

La technique de rechargement des plages a pour objectif de corriger le déficit sédimentaire d'une plage (Dean, 2002; Finkl, 2016). Le rechargement de la plage de Pilote Bar a présenté des résultats satisfaisants dans l'ensemble. Il a permis d'alimenter les plages voisines et de rehausser leur pente.

$\mathrm{Au}$ début on a noté une légère érosion de la plage et progressivement l'équilibre s'est rétabli. Les sédiments fins sont déplacés pas les courants vers le fond de la lagune. La sédimentologie est dominée par des grains grossiers $(0,78 \mathrm{~mm}$ à $0,219 \mathrm{~mm})$. Les échantillons sont généralement homogènes et bien classés et la distribution est presque symétrique. Les sediments ajoutés sur la haute plage sont redistribués en raison des conditions hydrodynamiques (Dean, 2002; Finkl, 2016).Tous les trauvaux similaires réalisés au $\mathrm{XX}^{\mathrm{e}}$ siècle ont montré un transfert de sédiments du haut de plage vers le bas de plage (Fichaut \& Suanez, 2008). A Sainte Flavie, le rechargement a entrainé l'augmentation initiale de la largeur de plage et du volume sédimentaire en 2012 (Bachand \& Comtois, 2016).

A Pilote Bar, le rechargement initié sur $700 \mathrm{~m}$ est resté stable et a, en même temps, permis à la plage au Nord du phare de s'engraisser à nouveau. La petite flèche joue le rôle de brise lame et maintient l'équilibre de la plage. Le suivi morpho dynamique depuis 2016, montre au début une légère érosion au Nord de la zone rechargée mais qui est vite stabilisée par l'évolution de la flèche. 
Du point de vue hydrodynamique, durant les périodes de vives eaux (coefficient 86 et 100) les vitesses de courants de marée dans la section du chenal sont inférieures à $2 \mathrm{~m} / \mathrm{s}$. Les vitesses des courants de flot semblent plus fortes que celles de jusant.

Les profils longshore présentent le même schéma d'évolution des vitesses de marée avec des valeurs moyennes plus faibles $(0,462 \mathrm{~m} / \mathrm{s}$ en période de flot ; 0,183 en période de jusant). Les profils mettent en évidence la présence d'un banc de sable de $200 \mathrm{~m}$ et qui semble favoriser la propagation des courants dans la partie sud du chenal. Les courants de faibles vitesses sont localisés au Nord du profil dans la partie lagunaire. Les profils cross-shore indiquent globalement des valeurs moins élevées que celles du profil longshore. Sur ce transect, les courants de flot restent plus élevés $(0,311 \mathrm{~m} / \mathrm{s})$ que ceux de jusant $(0,167 \mathrm{~m} / \mathrm{s})$. Les vitesses de courant mesurées en 2017 en période de mortes eaux (coefficient 50) présentaient déjà le même schéma d'évolution.

Globalement, on retrouve le schéma d'évolution de vitesse de courant d'un système côtier: des courants de flot forts et des courants de jusant faibles. Cependant, la présence de haut fond dans le chenal semble modifier la répartition des vitesses des courants favorisant le développement du système lagunaire avec des accrétions de sédiments fins dans les zones de faibles vitesses de courant. La technique de rechargement en sable tient compte de la réponse des plages aux conditions hydrodynamiques (Bernatchez et al., s. d.). La flèche bordant la lagune évolue vers le sud accompagnée d'une réduction de la profondeur de lagune. Ce contexte semble favoriser la stabilisation de la plage reconstituée de Pilote bar.

\section{Conclusion}

L'analyse des données recueillies entre 2016 et 2020 a permis de comprendre l'évolution morphologique de la plage rechargée de Gandiole. L'équilibre du profil de plage est contrôlé par les agents dynamiques qui déplacent les sédiments du haut de la plage vers le large. Cette tendance qui devrait déplacer le littoral vers le continent est ralentie par la mise en place de banc de sable. Leur accumulation a donné naissance à une flèche qui, progressivement a fini par isoler une lagune. Cette évolution qui se poursuit toujours continue de nous intéresser et sera l'objet de nos prochains travaux de recherche en utilisant les techniques modernes d'acquisition de données comme l'Aquadap et le drone. 


\section{References:}

1. Bâ, K., Wade, S., Niang, I., \& Trébossen, H. (2007). Cartographie radar en zone côtière à l'aide d'images multidates rso d'ers-2: application au suivi environnemental de la langue de barbarie et de l'estuaire du fleuve sénégal. 13.

2. Bachand, É., \& Comtois, S. (2016). Recharge en sable et revégétalisation de 2 plages de l'estuaire du Saint-Laurent, Québec. Le Naturaliste canadien, 140(2), 105-112. https://doi.org/10.7202/1036508ar

3. Bernatchez, P., \& Dubois, J.-M. M. (2006). Bilan des connaissances de la dynamique de l'érosion des côtes du Québec maritime laurentien. Géographie physique et Quaternaire, 58(1), 45-71. https://doi.org/10.7202/013110ar

4. Bernatchez, P., Fraser, C., Lefaivre, D., \& Dugas, S. (s. d.). Integrating anthropogenic factors, geomorphological indicators and local knowledge in the analysis of coastal flooding and erosion hazards. Ocean \&amp; Coastal Management, 54(8), 621-632. Consulté 14 janvier 2021, à l'adresse https://www.academia.edu/25816536/Integrating_anthropogenic_fact ors_geomorphological_indicators_and_local_knowledge_in_the_anal ysis_of_coastal_flooding_and_erosion_hazards

5. Bertin, X., \& Chaumillon, É. (2005). Apports de la modélisation sur bathymétries historiques dans la compréhension des évolutions des bancs de sable estuariens. Comptes Rendus Geoscience, 337(15), 1375-1383. https://doi.org/10.1016/j.crte.2005.06.007

6. Bindoff, N. L., Willebrand, J., Artale, V., Cazenave, A., Gregory, J. M., Gulev, S., Quéré, C. L., Levitus, S., Nojiri, Y., Shum, C. K., Talley, L. D., Unnikrishnan, A. S., Antonov, J., Curry, R., Emerson, S., Feely, R., Garcia, H., González-Davíla, M., Gruber, N., ... Wratt, D. (2007). Observations: Oceanic Climate Change and Sea Level. 49.

7. Chaumillon, E., Wöppelmann, G., Karpytchev, M., \& Bertin, X. (2011). Mesures et modélisations des évolutions du niveau marin, des vagues, des tempêtes et des évolutions des littoraux pour une gestion durable des littoraux. VertigO, Hors-série 9. https://doi.org/10.4000/vertigo.10947

8. Dean, R. G. (2002). Beach Nourishment: Theory and Practice. World Scientific.

9. DEEC. (2015). Specifications techniques et conditions de bonne excution-Termes de Reference (T.D.R), Direction de l'Environnement et des Etablissements Classés, 2015, 18p.

10. Durand, P., Anselme, B., \& Thomas, Y.-F. (2010). L'impact de l'ouverture de la brèche dans la langue de Barbarie à Saint-Louis du 
Sénégal en 2003 : Un changement de nature de l'aléa inondation? Cybergeo: European Journal of Geography. https://doi.org/10.4000/cybergeo.23017

11. Faye, I. (2010). Dynamique du trait de côte sur les littoraux sableux de la Mauritanie à la Guinée-Bissau (Afrique de l'Ouest) : Approches régionale et locale par photo-interprétation, traitement d'images et analyse de cartes anciennes. 322.

12. Fichaut, B., \& Suanez, S. (2008). Le rechargement de la plage de Sainte-Anne-du-Portzic (Brest-Finistère): Quand la recherche fondamentale se met au service d'une gestion cohérente du littoral. Norois, 208, 29-44. https://doi.org/10.4000/norois.2175

13. Finkl, C. W. (2016). Beach Renourishment by Eric Bird and Nick Lewis. Journal of Coastal Research, 32(4), 998-998. https://doi.org/10.2112/JCOASTRES-D-16A-00004.1

14. Gac, J.-Y., Kane, A., \& Monteillet, J. (1982). Migrations de l'embouchure du fleuve Sénégal depuis 1850. 4.

15. Hamm, L., Capobianco, M., Dette, H. H., Lechuga, A., Spanhoff, R., \& Stive, M. J. F. (2002). A summary of European experience with shore nourishment. Coastal Engineering, 47(2), 237-264. https://doi.org/10.1016/S0378-3839(02)00127-8

16. Harley, M. D., Andriolo, U., Armaroli, C., \& Ciavola, P. (2014). Shoreline rotation and response to nourishment of a gravel embayed beach using a low-cost video monitoring technique: San MicheleSassi Neri, Central Italy. Journal of Coastal Conservation, 18(5), 551-565. https://doi.org/10.1007/s11852-013-0292-x

17. Jeanson, M., Dolique, F., \& Anthony, E. J. (2011). Un réseau de surveillance des littoraux face au changement climatique en milieu insulaire tropical: L'exemple de Mayotte. VertigO, Volume 10 Numéro 3. https://doi.org/10.4000/vertigo.10512

18. Mason, M. A. (1949). Erosion et protection des cotes. La Houille Blanche, 1, 14-37. https://doi.org/10.1051/lhb/1949015

19. Newman, D. E. (1976). Beach replenishment: Sea defenses and a review of the artificial beach replenishment (Vol. 6).

20. Nicholls, R. J. (1998). Assessing erosion of sandy beaches due to sealevel rise. Geological Society, London, Engineering Geology Special Publications, $15(1)$, 71-76. https://doi.org/10.1144/GSL.ENG.1998.015.01.08

21. Observatoire National sur les Effets du Réchauffement Climatique (Éd.). (2015). Le littoral dans le contexte du changement climatique: Rapport au Premier ministre et au Parlement. La Documentation française.

22. Paskoff, R. (2007). La crise des plages : pénurie de sédiments. 5. 
23. Rogers, S. M. (2000). Beach nourishment for hurricane protection: NORTH CAROLINA PROJECT PERFORMANCE IN HURRICANES DENNIS AND FLOYD. 6.

24. Solomon, S., \& IPCC (Éds.). (2007). Climate change 2007: The physical science basis; contribution of Working Group I to the Fourth Assessment Report of the Intergovernmental Panel on Climate Change (1st published). UNEP.

25. Sy, B. A. (s. d.). L'ouverture de la brèche de la Langue de Barbarie et ses conséquences, Approche géomorphologique | Recherches Africaines. Consulté 24 août 2020, à l'adresse https://revues.ml/index.php/recherches/article/view/802

26. Sy, Boubou Aldiouma, Bilbao, I. A., Sy, A. A., Perez, I. S., \& Valido, S. R. (2013). Résultats du suivi 2010-2012 de l'évolution de la brèche ouverte sur la Langue de Barbarie au Sénégal et de ses conséquences. Physio-Géo, Volume 7, 223-242. https://doi.org/10.4000/physiogeo.3569

27. Trinh, P. T., Tan, M. T., \& Ozer, A. (2007). Évolution de la zone côtière de Hai Phong au Nord du Vietnam. 8. 